\title{
BMJ Open Protocol for a validation study of the translated stapesplasty outcome test 25 for measurement of disease-specific quality of life in Dutch patients with otosclerosis
}

\author{
Esther E Blijleven, Hans G X M Thomeer, Robert Stokroos, Inge Wegner (C)
}

To cite: Blijleven EE, Thomeer HGXM, Stokroos R, et al. Protocol for a validation study of the translated stapesplasty outcome test 25 for measurement of disease-specific quality of life in Dutch patients with otosclerosis. BMJ Open 2019;9:e030219. doi:10.1136/ bmjopen-2019-030219

- Prepublication history for this paper is available online. To view these files, please visit the journal online (http://dx.doi. org/10.1136/bmjopen-2019030219).

Received 04 March 2019 Revised 25 November 2019 Accepted 26 November 2019

Check for updates

(C) Author(s) (or their employer(s)) 2019. Re-use permitted under CC BY-NC. No commercial re-use. See rights and permissions. Published by BMJ.

Department of Otorhinolaryngology - Head and Neck Surgery, University Medical Center Utrecht, Utrecht, The Netherlands

Correspondence to Dr Inge Wegner;

I.Wegner@umcutrecht.nl

\section{ABSTRACT}

Introduction Otosclerosis is a common cause of acquired conductive hearing loss and can be treated using hearing aids or surgically. Surgical success rates or surgical results are usually reported using pure-tone audiometric thresholds and/or speech discrimination scores. Audiometric results and patient-reported quality of life after stapes surgery do not seem to correlate well. It is therefore our opinion that health-related quality of life measurements should be implemented as an additional outcome measure after stapes surgery. So far, there is a lack of a valid, reliable and clinically feasible measuring tool for determining health-related quality of life in Dutch patients with otosclerosis who undergo stapes surgery.

Methods and analysis A prospective validation study was designed to translate and validate the diseasespecific Stapesplasty Outcome Test 25 (SPOT-25) in a population of Dutch patients with otosclerosis who undergo primary stapes surgery. A total of 125 patients with otosclerosis who will be undergoing primary stapes surgery and 50 healthy controls will be included. The patients with otosclerosis will fulfil several questionnaires preoperatively, 6 to 8 weeks postoperatively and 8 to 10 weeks postoperatively with a 2-week interval between the postoperative administrations. The patients' audiometric results, which are measured routinely before and after undergoing primary stapes surgery, will also be used. The healthy controls will fulfil the translated SPOT-25 once. First, the original SPOT-25 will be translated from German to Dutch in a six-step process. Second, the translated SPOT-25 will be pilot-tested in a subset of patients. Lastly, validity, reliability and responsiveness of the translated SPOT-25 will be analysed.

Ethics and dissemination The research protocol was approved by the Institutional Review Board of the University Medical Center Utrecht (protocol 18-768/C; V.1, November 2018). Initially, we planned to include 50 patients. At a later stage we decided to increase the sample size to 100 patients. We notified the Institutional Review Board of this change to the protocol. The trial results will be disseminated through peer-reviewed medical journals and presented at scientific conferences. Trial registration number NL7586.

\section{Strengths and limitations of this study}

- This study aims to translate the German Stapesplasty Outcome Test 25 (SPOT-25) to the Dutch language and validate the translated SPOT-25 in a Dutch population of patients with otosclerosis undergoing primary stapes surgery.

- This study is the first to validate a patient-reported questionnaire that evaluates health-related quality of life in Dutch patients with otosclerosis.

- The findings of this trial could improve our otosclerosis research and clinical practice because we will be able to take into account health-related quality of life in patients with otosclerosis following primary stapes surgery when deciding on what surgical techniques to use.

\section{INTRODUCTION}

Otosclerosis is characterised by abnormal bone growth in the middle ear that may cause fixation of the stapes footplate, resulting in progressive conductive hearing loss, vertigo and/or tinnitus. ${ }^{1}$ The conductive hearing loss can be treated using hearing aids or surgically in a procedure called stapes surgery. Stapes surgery includes both stapedotomy and stapedectomy. In stapedotomy, the stapes footplate is fenestrated and a prosthesis is inserted in the fenestration. In stapedectomy, (part of) the stapes footplate is removed and a prosthesis is replaced by a prosthesis.

In 1995 and 2012, the American Academy of Otolaryngology-Head and Neck Surgery published guidelines on minimal reporting standards for evaluating hearing outcomes in conductive hearing loss. ${ }^{2} 3$ Both guidelines recommend the use of pure-tone audiometric thresholds and/or speech discrimination scores which both have traditionally been used to report postoperative hearing results. These measures are useful 
in assessing surgical success, defined as a postoperative air-bone gap of $10 \mathrm{~dB}$ or less, and (monaural) ability to detect sounds or speech. Surgeons generally assume that these outcomes reflect the patient's subjective improvement and health-related quality of life, that is, an individual's perception of how an illness and its treatment affect the physical, mental and social aspects of his or her life. ${ }^{4}$ However, studies show that audiometric results do not correlate well with health-related quality of life in patients with otosclerosis after stapes surgery. ${ }^{56}$ Both studies show that primary stapes surgery is associated with an improvement in health-related quality of life measured by generic and hearing-specific questionnaires. Correlation analyses of hearing ability and quality of life measurements show that the degree of postoperative airbone gap, change in air-bone gap and tinnitus do not correlate with any of the scores from these generic quality of life questionnaires and hearing-specific quality of life questionnaires. ${ }^{56}$ Furthermore, patient-reported hearing disability correlates better with health-related quality of life than with audiometric results. ${ }^{56}$ The latter emphasises the need for patient-reported outcome measures in determining surgical success in patients with otosclerosis. Patient-reported outcome measures are used in clinical practice and research to gain insight from the patient's perspective regarding their disability symptoms and how their disability and its treatment affect their quality of life. ${ }^{7}$ Patient-reported outcome measures include generic and disease-specific questionnaires of quality of life.

A systematic review concerning hearing outcome measures demonstrated that more than 50 different patient-reported questionnaires have been used in 122 studies researching hearing loss in adults. ${ }^{8}$ None of these questionnaires have been designed to specifically measure health-related quality of life in patients with otosclerosis nor have these questionnaires been validated in a population of patients with otosclerosis. There is one questionnaire not mentioned in this review that has been validated in a population of patients with otosclerosis undergoing stapes surgery: the Stapesplasty Outcome Test 25 (SPOT-25) ${ }^{9}$ The SPOT-25 has been validated for use in the German language and culture only. We aim to translate the SPOT-25 to the Dutch language and validate the translated SPOT-25 in a Dutch population of patients with otosclerosis undergoing primary stapes surgery. Translation of the SPOT-25 to Dutch is not sufficient on its own, because the questionnaire may also have to be adapted culturally in order to adequately reflect healthrelated quality of life in patients with otosclerosis in the Netherlands. ${ }^{40}$ Some items that were relevant in the German culture may be irrelevant in the Dutch culture.

\section{Methods and analysis}

\section{Study design}

The study design will be a prospective validation study. First, the original SPOT-25 will be translated from German to Dutch in a six-step process. Second, the translated SPOT-25 will be validated. The study will take 2 years to complete. The translation process was started in December 2018. The expected completion date for data collection is December 2020.

\section{Sample selection}

Three samples are required to achieve the study objectives: two samples of Dutch patients with otosclerosis who will undergo primary stapes surgery and one sample of healthy controls from the general population.

In order to be eligible for inclusion in this study, the patients with otosclerosis will have to meet the following inclusion and exclusion criteria.

\section{Inclusion criteria}

- Age $\geq 18$.

- Otosclerosis based on a clinical history of progressive hearing loss and pure-tone audiometry showing a conductive hearing loss with an air-bone gap of $15 \mathrm{~dB}$ nHL or more in the range of 500, 1000, 2000 and $4000 \mathrm{~Hz}$

- Scheduled or on the waiting list for primary stapes surgery;

- Willingness and ability to fulfil the questionnaires outlined in the research protocol;

- Good understanding of the Dutch language confirmed by questioning the patients. Patients should be proficient users of the Dutch language, meaning they are either native speakers or they can understand everything heard or read with ease and express themselves fluently and precisely (equivalent to Common European Framework of Reference for Languages(CEFR) level C2).

\section{Exclusion criteria}

- Scheduled or on the waiting list for revision stapes surgery.

- Disability that could interfere with audiometric evaluation and/or questionnaire fulfilment.

In order to be eligible for inclusion in this study, the healthy controls will have to meet the following inclusion and exclusion criteria:

\section{Inclusion criteria}

- Age between 30 and 60 years

- Willingness and ability to fulfil the questionnaires outlined in the research protocol;

- Good understanding of the Dutch language confirmed by questioning the healthy controls. Healthy controls should be proficient users of the Dutch language, meaning they are either native speakers or they can understand everything heard or read with ease and express themselves fluently and precisely (equivalent to CEFR level C2).

\section{Exclusion criteria}

- A history of hearing loss, ear disease (with the exception of uncomplicated acute otitis or otitis media with effusion in childhood) or previous middle ear surgery 
(with the exception of the placement of ventilation tubes in childhood) based on questioning the healthy control subject.

- Disability that could interfere with questionnaire fulfilment.

The age range for the control group is different from the age range in the patient group. We want to make sure that the age of the included controls is approximately the same as that of the included patients. Based on three large cohort studies, patients with otosclerosis are on average 39 to 47 years old when they undergo primary stapes surgery with an SD of 11 to 15 years. ${ }^{11-13}$ Therefore, we choose to include controls in the age range 30 to 60 years.

\section{Sample size}

A total of 15 patients will be included to pilot-test the translated SPOT-25, and a further 100 patients will be included to validate the translated SPOT-25. For construct validation studies and responsiveness studies in which correlation coefficients are calculated and reliability studies, a minimum of 50 patients is recommended. ${ }^{44}$ For cross-cultural validation/assessment of measurement invariance using confirmatory factor analysis, a minimum of 100 patients is recommended. ${ }^{414}$ To anticipate withdrawal of $10 \%$ of participants, we will include an extra 10 participants for a total of 125 patients. A total of 50 healthy controls will be included. At the ear, nose and throat (ENT) department at the University Medical Center Utrecht, 80 patients with otosclerosis undergo primary stapes surgery per year. Assuming a participation rate of $80 \%$, we expect to be able to include 125 patients with otosclerosis and 50 healthy controls in 24 months' time.

Patients will be recruited from the outpatient ENT department at the University Medical Center Utrecht. Eligible patients will be informed about the study by their treating physician. These patients have already given their consent for primary stapes surgery. The treating physician will provide the patient with an information letter and informed consent form. Healthy controls will be recruited through posters that will be placed in the University Medical Center Utrecht. After contacting the researchers, the healthy controls will receive an information letter and informed consent form. Patients and healthy controls consent to the use of their data for the research purposes outlined in this protocol which includes publication of the results once the trial has been completed. Patients and health controls will not receive compensation for participation in the trial.

\section{Outcomes to be measured}

Validation of the translated SPOT-25 will entail three measurement moments in the samples of Dutch patients with otosclerosis: preoperatively, 6 to 8 weeks postoperatively and 8 to 10 weeks postoperatively. There is a 2-week time window for the postoperative measurements because in clinical practice the first postoperative follow-up appointment is scheduled within this 2-week time window, and there will be a 2-week interval between the two postoperative measurements moments. The translated SPOT-25 and the Glasgow Health Status Questionnaire (GHSQ) will be fulfilled preoperatively during the patients' preoperative visit to the outpatient department. The translated SPOT-25, the GHSQ and the Glasgow Benefit Inventory (GBI) will be fulfilled 6 to 8 weeks postoperatively during the patients' postoperative visit to the outpatient department. The translated SPOT-25 will be fulfilled 8 to 10 weeks postoperatively. This last measurement will take place at home. The questionnaire will be sent by post. We are aware that the different test setting is a potential confounder. However, having the last measurement take place at home will limit loss to follow-up. Furthermore, the patients' audiometric results, which are measured routinely in every patient before and after undergoing primary stapes surgery, will be used. Audiometric testing will take place on the same day that the patients complete the questionnaires.

Validation of the translated SPOT-25 will entail only one measurement moment in the sample of healthy controls. They will fulfil the translated SPOT-25 once. The questionnaire will be sent to the healthy controls by post.

\section{Audiometric results}

Pure-tone audiometric thresholds and speech discrimination scores are routinely obtained preoperatively and 6 to 8 weeks after primary stapes surgery in our otosclerosis patients treated with primary stapes surgery. We will be using these results for our study, but these do not require extra efforts from the included patients. Audiologic parameters that we will assess include air-conduction thresholds, bone-conduction thresholds and air-bone gaps at $500,1000,2000$ and $4000 \mathrm{~Hz}$ as well as speech discrimination scores.

\section{Health-related quality of life}

Health-related quality of life will be assessed using the following questionnaires:

- Translated SPOT-25: a 25-item questionnaire measuring health-related quality of life in patients with otosclerosis. ${ }^{9}$ Four domains ('hearing function', 'tinnitus', 'mental condition' and 'social restrictions'), as well as one item focusing on the general impact of otosclerosis on health-related quality of life, are measured based on a five-point Likert scale. The total score ranges from 0 (slightest restriction in health-related quality of life) to 100 points (the biggest restriction in health-related quality of life).

- GHSQ: an 18-item questionnaire measuring the effect of an otologic problem on quality of life at the time the questionnaire is fulfilled. Three domains ('general', 'social support' and 'physical health') are measured based on a five-point Likert scale ranging from high health status to low health status. The total score ranges from 0 to 100 points; 
- GBI: an 18-item questionnaire measuring the change in health status as a result of a surgical intervention. A specific version of the GBI will be used that has been validated to measure changes in health status as a result of otorhinolaryngological procedures. ${ }^{15}$ The same three domains as the GHSQ are measured based on the same five-point Likert scale. The total score ranges from -100 (maximum negative benefit), through 0 (no benefit), to +100 points (maximum benefit).

The GHSQ and GBI were chosen as comparator patientreported outcome measures because they are most often used in our daily clinical practice and they most closely resemble the (translated) SPOT-25.

\section{Data analysis plan}

Translation process

First, the original SPOT-25 will be translated from German to Dutch in a six-step process ${ }^{10}$ :

1. Forward translation: two bilingual translators with the Dutch language as the mother tongue will translate the questionnaire from German to Dutch.

2. The forward translations will be combined into one translation.

3. Backward translation: two bilingual translators with the German language as the mother tongue will translate the forward-translated questionnaire back from Dutch to German. The translators will be blinded to the original German version of the SPOT-25.

4. An expert committee of the four translators and the researchers will review all translations and will compose a final version.

5. The final version will be tested in a small sample of the target population of 15 patients with otosclerosis for pilot-testing.

6. The developers of the original SPOT-25 will appraise the final version and adaptation process.

\section{Content validity}

Content validity is the degree to which the content of a measurement instrument is an adequate reflection of the construct to be measured. ${ }^{4}$ The translated SPOT-25 will be pilot-tested for content validity in a set of 15 patients with otosclerosis. Data from the pilot will be analysed to confirm the face validity of the translated SPOT-25. Face validity is the degree to which a measurement instrument looks as though it is an adequate reflection of the construct to be measured; in this case, the degree to which the translated SPOT-25 is an adequate reflection of health-related quality of life in patients with otosclerosis in this study. ${ }^{4}$ It is a subjective assessment and cannot be quantified. We will carry out interviews. Patients will be asked about the comprehensibility and comprehensiveness of the translated SPOT-25. One of the researchers (EEB) will conduct the interviews. The interviews will be transcribed verbatim. Two researchers (EEB and IW) will analyse the data. All items will be tested in their final form which means that the translated SPOT-25 will be adapted and re-tested if necessary in a new set of 15 patients with otosclerosis.

\section{Construct validity using hypotheses testing}

Construct validity is the degree to which the scores of a measurement instrument, in this case the translated SPOT-25, are consistent with hypotheses, for example, with regard to internal relationships, relationships with scores of other instruments or differences between relevant groups. ${ }^{4}$ Hypotheses will be formulated regarding the relationship between the translated SPOT-25 and other measurement instruments that measure healthrelated quality of life in patients with otologic problems or diseases. The translated SPOT-25 will be compared with the GBI, GHSQ and audiometric results. We expect (domains of) the translated SPOT-25 to correlate with all three measurement instruments. We expect the total score of the translated SPOT-25 to have at least a 0.1 higher correlation with the postoperative GHSQ than with the GBI and at least a 0.2 higher correlation with the postoperative GHSQ than the postoperative air conduction, postoperative air-bone gap, gain in air-bone gap and success defined as air-bone gap closure $10 \mathrm{~dB}$ or less. We expect the 'hearing function' domain of the translated SPOT-25 to have at least a 0.1 higher correlation with the GBI than with the GHSQ. Furthermore, we expect the 'hearing function' domain to have at least a 0.1 higher correlation with gain in air conduction than with postoperative air conduction and at least a 0.2 higher correlation with gain in air conduction than with postoperative air-bone gap, gain in air-bone gap and success defined as air-bone gap closure to $10 \mathrm{~dB}$ or less. We expect weak correlations ( 0.1 to 0.4$)$ between the other three domains ('tinnitus', 'mental condition' and 'social restrictions') and the postoperative air-bone gap, gain in air-bone gap and success. We expect the 'mental condition' and 'social restrictions' domains of the translated SPOT-25 to have at least a 0.3 higher correlation with the GHSQ than with the GBI. The above-mentioned hypotheses will be tested using Pearson correlation coefficients.

\section{Cross-cultural validation using measurement invariance}

If a measurement instrument performs in exactly the same way in different populations, we speak of measurement invariance. We will test for measurement invariance using confirmatory factor analysis. We hypothesise that the original four-factor model ('hearing function', 'tinnitus', 'mental condition' and 'social restrictions') has an adequate fit in our population of patients with otosclerosis and performs well in comparison with other possible factor solutions. We will test a one-factor model, a twofactor model of hearing function/tinnitus and mental/ social factors and a three-factor model of hearing function/tinnitus, mental factors and social factors. We will use the comparative fit index, the root mean square error of approximation and the standardised root mean square residual to assess the fit of the models to the data. ${ }^{4}$ Models with a comparative fit close to 0.95 or higher, root mean 
square error of approximation close to 0.06 or lower and root mean square residual close to 0.08 or lower are representative of good-fitting models. ${ }^{4}$

We expect our sample to be similar to the sample from the original German validation study. We will use the same inclusion criteria: patients with otosclerosis based on a clinical history of progressive hearing loss and puretone audiometry showing a conductive hearing loss with an air-bone gap of $15 \mathrm{~dB} \mathrm{nHL}$ or more in the range of $500,1000,2000$ and $4000 \mathrm{~Hz}$ who will undergo primary stapes surgery. ${ }^{9}$

\section{Discriminative validity}

For assessment of discriminative validity, the preoperative total score and subscores of the translated SPOT-25 in patients with otosclerosis will be compared with the total score and subscores of the translated SPOT-25 in the healthy control group using Pearson correlation coefficients.

\section{Reliability}

Reliability is the extent to which scores for patients who have not changed are the same for repeated measurement under several conditions, for example, using different sets of items from the same multi-item measurement instrument (internal consistency) or over time (test-retest). ${ }^{4}$ In our study, we are interested in the degree to which different sets of items from the translated SPOT-25 measure health-related quality of life in patients with otosclerosis (internal consistency) and the degree to which SPOT-25 scores change over time (test-retest). Internal consistency of the total score and the subscores of the translated SPOT-25 will be measured by calculating Cronbach's alpha. Acceptable values of Cronbach's alpha will be between 0.70 and $0.90 .{ }^{4}$ Test-retest reliability will be assessed using two-way random effects model of intraclass correlation coefficient (ICC). An ICC of 0.70 will be considered acceptable. ${ }^{4}$ The data of the two postoperative measurements will be used. Patients have been shown to be stable with regard to audiometric tests over time. ${ }^{11}$

\section{Responsiveness}

Responsiveness is the ability of an instrument to detect change over time in the construct to be measured; in this case, the ability of the translated SPOT-25 to detect change in health-related quality of life in patients with otosclerosis. Since there is no gold standard available in detecting change in health-related quality of life in patients with otosclerosis, we will resort to testing hypotheses. ${ }^{4}$ The change scores of the translated SPOT-25 will be compared with the change scores of the GHSQ and audiometric results. We expect the correlation of change on the translated SPOT-25 with change on the GHSQ to be at least 0.1 higher than the correlation of change on the translated SPOT-25 with gain in air conduction and at least 0.2 higher than the correlation of change on the translated SPOT-25 with gain in air-bone gap. We expect the correlation of change on the translated SPOT-25 with gain in air conduction to be at least 0.2 higher than the correlation of change on the translated SPOT-25 with gain in air-bone gap. We expect the correlation of change in the 'hearing function' domain of the translated SPOT-25 with gain in air conduction to be at least 0.2 higher than the correlation of change on the translated SPOT-25 with gain in air-bone gap. We expect weak correlations (0.1 to 0.4$)$ between change in the other three domains ('tinnitus', 'mental condition' and 'social restriction') and the gain in air-bone gap. The above-mentioned hypotheses will be tested using Pearson correlation coefficients.

\section{Missing data}

We will report the percentage of missing values and investigate reasons for missing data. Missing data will be imputed. Furthermore, we will consider deleting incidental items with a large percentage of missing data. The decision will be based on the weighting between the percentage of missing data and the importance of that specific item. We will regard more than $15 \%$ missing data in one item as not acceptable.

\section{Patient and public involvement}

Patients and the public were not involved in the design, recruitment and conduct of the study. The results will be disseminated to study participants by post or by email based on their preferences.

\section{ETHICS AND DISSEMINATION}

The study will be conducted according to the principles of the Declaration of Helsinki and 'gedragscode gezondheidsonderzoek' en 'toetsingscriteria eenvormige toetsing' that consist of the laws: 'WGBO' (Wet op de Geneeskundige Behandelings Overeenkomst) and 'AVG' (Algemene Verordening Gegevensbescherming).

All data will be handled confidentially. The data will be coded by using a unique patient identification number. Results will be entered into IBM SPSS statistics V.24 software for statistical analysis and stored on the investigators' computer which is secured by a password and located in a locked room. The research data will be archived on the research network disc of the division for 15 years after the study has ended.

The trial results will be disseminated through peerreviewed medical journals and presented at scientific conferences.

\section{TRIAL STATUS}

The trial is currently in the recruitment phase.

Contributors EEB: executive investigator, developing protocol, drafting and revising manuscript, approval of final version. HGXT: surgeon, initial idea, developing protocol, revising manuscript, approval of final version. RS: surgeon, revising manuscript, approval of final version. IW: initial idea, developing protocol, drafting and revising manuscript, approval of final version.

Funding The authors have not declared a specific grant for this research from any funding agency in the public, commercial or not-for-profit sectors. 
Competing interests None declared.

Patient consent for publication Not required.

Ethics approval The research protocol was approved by the Institutional Review Board of the University Medical Center Utrecht (protocol 18-768/C; V.1, November 2018).

Provenance and peer review Not commissioned; externally peer reviewed.

Open access This is an open access article distributed in accordance with the Creative Commons Attribution Non Commercial (CC BY-NC 4.0) license, which permits others to distribute, remix, adapt, build upon this work non-commercially, and license their derivative works on different terms, provided the original work is properly cited, appropriate credit is given, any changes made indicated, and the use is non-commercial. See: http://creativecommons.org/licenses/by-nc/4.0/.

ORCID iD

Inge Wegner http://orcid.org/0000-0002-4124-7224

\section{REFERENCES}

1 Menger DJ, Tange RA. The aetiology of otosclerosis: a review of the literature. Clin Otolaryngol Allied Sci 2003;28:112-20.

2 American Academy of Otolaryngology - Head Neck Surgery Foundation, Inc. Committee on hearing and equilibrium guidelines for the evaluation of results of treatment of conductive hearing loss. AmericanAcademy of otolaryngology-head and neck surgery Ffoundation, Inc. Otolaryngol Head Neck Surg 1995;113:186-7.

3 Gurgel RK, Jackler RK, Dobie RA, et al. A new standardized format for reporting hearing outcome in clinical trials. Otolaryngol Head Neck Surg 2012;147:803-7.
4 de Vet HCW, Terwee CB, Mokkink LB, et al. Measurement in medicine: a practical guide. Cambridge: Cambridge Press, 2011.

5 Redfors YD, Olaison S, Karlsson J, et al. Hearing-related, healthrelated quality of life in patients who have undergone otosclerosis surgery: a long-term follow-up study. Int J Audiol 2015;54:63-9.

6 Subramaniam K, Eikelboom RH, Marino R, et al. Patient's quality of life and hearing outcomes after stapes surgery. Clin Otolaryngol 2006;31:273-9.

7 Black N. Patient reported outcome measures could help transform healthcare. BMJ 2013;346:f167.

8 Granberg S, Dahlström J, Möller C, et al. The ICF Core Sets for hearing loss--researcher perspective. Part I: Systematic review of outcome measures identified in audiological research. Int $J$ Audiol 2014;53:65-76.

9 Lailach S, Schenke T, Baumann I, et al. [Development and validation of the Stapesplasty Outcome Test 25 (SPOT-25)]. HNO 2017;65:973-80.

10 Beaton DE, Bombardier C, Guillemin F, et al. Guidelines for the process of cross-cultural adaptation of self-report measures. Spine 2000;25:3186-91.

11 Vincent R, Sperling NM, Oates J, et al. Surgical findings and longterm hearing results in 3,050 stapedotomies for primary otosclerosis: a prospective study with the otology-neurotology database. Otol Neurotol 2006;27:S25-47.

12 Kisilevsky VE, Dutt SN, Bailie NA, et al. Hearing results of 1145 stapedotomies evaluated with Amsterdam hearing evaluation plots. $J$ Laryngol Otol 2009;123:730-6.

13 Bittermann AJN, Rovers MM, Tange RA, et al. Primary stapes surgery in patients with otosclerosis: prediction of postoperative outcome. Arch Otolaryngol Head Neck Surg 2011;137:780-4.

14 Terwee CB. COSMIN checklist with 4-point scale. Available: www. cosmin.nl [Accessed Jun 2018].

15 Robinson K, Gatehouse S, Browning GG. Measuring patient benefit from otorhinolaryngological surgery and therapy. Ann Otol Rhinol Laryngol 1996;105:415-22. 